\title{
Effect of Integrated Use of Organic and Inorganic Sources of Nutrients and Biofertilizers on Growth and Nutrient Content of Maize (Zea mays L.)
}

\author{
P. Venkata Subbaiah ${ }^{1 *}$ and A. Sai Ram $^{2}$ \\ ${ }^{1}$ Department of Soil Science \& Agricultural Chemistry, Agricultural College, \\ Bapatla, Andhra Pradesh-522101, India \\ ${ }^{2}$ Soil Test Crop Response, Agricultural Research Institute, Hyderabad-30, India \\ *Corresponding author
}

\begin{abstract}
A B S T R A C T

\begin{tabular}{|l|}
\hline Ke y w o r d s \\
Maize growth, \\
Nutrient content, \\
Nutrient \\
management
\end{tabular}
\end{abstract}

\section{Introduction}

Maize is known as "queen of cereals" due to its higher production potential. It is established that maize is nutrient exhaustive crop and fertilizer use is probably the most crucial input next only to irrigation since as high as 120 to $250 \mathrm{~kg} \mathrm{~N} \mathrm{ha}^{-1}$ (Srikanth et al., 2009, Panwar and Munda, 2006 and Anitha et al., 2007), 26.4 to $125 \mathrm{~kg} \mathrm{P}^{-1}$ (Verma et al., 2003 and Srikanth et al., 2009) has been reported to be applied to maximize productions. Integrated use of fertilizers and organic manures help in maintaining yield stability in most of the agro-ecosystems through correction of secondary and micro nutrient deficiencies, enhancing the efficiency of applied nutrients and providing favourable soil physical conditions. Integrated nutrient is more efficient than other management practices.

\section{Materials and Methods}

A field experiment was conducted during kharif (Maize) on Alfisols at College Farm, College of Agriculture, Rajendranagar, Hyderabad. The experimental soil was sandy loam, neutral in reaction ( $\mathrm{pH} 7.28$ ), non saline (EC $0.22 \mathrm{dSm}^{-1}$ ), low in organic carbon 
$(0.49 \%)$, low in alkaline $\mathrm{KMNO}_{4}$ extractable $\mathrm{N}\left(186 \mathrm{~kg} \mathrm{ha}^{-1}\right)$, medium in available $\mathrm{P}(23.27$ $\left.\mathrm{kg} \mathrm{ha}^{-1}\right)$ and high in available $\mathrm{K}\left(395 \mathrm{~kg} \mathrm{ha}^{-1}\right)$. The experiment was laid out in Randomized Block Design consisting of twelve treatment combinations each replicated thrice. The treatments consisted control $\left(\mathrm{T}_{1}\right)$; three inorganic $\mathrm{N}$ and $\mathrm{P}$ levels namely $50 \% \mathrm{~N}$ and $\mathrm{P}$ through $\mathrm{RDF}\left(\mathrm{T}_{2}\right), 75 \% \mathrm{~N}$ and $\mathrm{P}$ through $\operatorname{RDF}\left(\mathrm{T}_{3}\right)$ and $100 \% \mathrm{~N}$ and $\mathrm{P}$ through $\mathrm{RDF}\left(\mathrm{T}_{4}\right)$ and integrated nutrient management treatments namely $75 \% \mathrm{~N}$ through $\mathrm{RDF}+$ $25 \% \mathrm{~N}$ through poultry manure $\left(\mathrm{T}_{5}\right), 75 \% \mathrm{~N}$ through $\mathrm{RDF}+25 \% \mathrm{~N}$ through poultry manure + azotobacter $\left(\mathrm{T}_{6}\right), 75 \% \mathrm{~N}$ through $\mathrm{RDF}+25 \% \mathrm{~N}$ through vermicompost $\left(\mathrm{T}_{7}\right)$, $75 \% \mathrm{~N}$ through $\mathrm{RDF}+25 \% \mathrm{~N}$ through vermicompost + azotobacter $\left(\mathrm{T}_{8}\right), 75 \% \mathrm{P}$ through $\mathrm{RDF}+25 \% \mathrm{P}$ through poultry manure $\left(\mathrm{T}_{9}\right), 75 \% \mathrm{P}$ through $\mathrm{RDF}+25 \% \mathrm{P}$ through poultry manure + phosphorus solubulising bacteria $\left(\mathrm{T}_{10}\right), 75 \% \mathrm{P}$ through $\mathrm{RDF}+25 \% \mathrm{P}$ through vermicompost $\left(\mathrm{T}_{11}\right)$, $75 \% \mathrm{P}$ through $\mathrm{RDF}+25 \% \mathrm{P}$ through vermicompost + phosphorus solubulising bacteria $\left(\mathrm{T}_{12}\right)$.

The organic sources and biofertilizers were applied at the time of field preparation. Popular variety viz., DHM-111(Maize) raised in the field with a spacing of $60 \times 20 \mathrm{~cm}$ (Maize) and all the recommended cultural practices were fallowed. The maize crop was harvested at maturity i.e. at 100 days after sowing (DAS).

\section{Results and Discussion}

Effect of different fertility management treatments on grain yield, concentration of $\mathrm{N}, \mathrm{P}, \mathrm{K}, \mathrm{S}, \mathrm{Fe}, \mathrm{Mn}, \mathrm{Zn}$ and $\mathrm{Cu}$ and their uptake by maize at flowering stage

The nutrient content in the plant sample at flowering stage in the present investigation (Table 1 and 2) showed that the concentration of $\mathrm{N}$ and $\mathrm{P}$ increased significantly on application of recommended level of $120 \mathrm{~kg}$ $\mathrm{N}$ and $60 \mathrm{~kg} \mathrm{P}_{2} \mathrm{O}_{5} \mathrm{ha}^{-1}$ compared to control and reduction in the level of these nutrients to $50 \%$. The micronutrients viz., $\mathrm{Fe}, \mathrm{Zn}$ and $\mathrm{Cu}$ concentration was also significantly higher in maize fertilized with the recommended level of $\mathrm{N}$ and $\mathrm{P}$. The crop in turn responded to produce abundant dry matter of $52.13 \mathrm{q} \mathrm{ha}^{-1}$ because of the larger concentration of these nutrients. The dry matter content was merely $30 \mathrm{q} \mathrm{ha}^{-1}$ in the unfertilized crop. The uptake of $\mathrm{N}, \mathrm{P}, \mathrm{K}, \mathrm{S}, \mathrm{Fe}, \mathrm{Mn}, \mathrm{Zn}$ and $\mathrm{Cu}$ showed a tremendous increase with increase in the level of $\mathrm{N}$ and $\mathrm{P}$ fertilizers with incremental additions up to the recommended level of $\mathrm{N}$ and $\mathrm{P}$ fertilizers. The integrated nutrient management treatments scheduled to substitute $25 \% \mathrm{~N}$ through poultry manure or vermicompost or $25 \% \mathrm{P}$ through these organic sources with or without the addition of biofertilzers viz., Azotobacter and phosphorus solubilising bacteria significantly increased the concentration of $\mathrm{P}, \mathrm{Fe}, \mathrm{Mn}$ and $\mathrm{Cu}$ over the crop treated with inorganic source of nutrients. However there was no significant improvement in the dry matter content. The uptake of $\mathrm{N}, \mathrm{P}, \mathrm{Mn}$ and $\mathrm{Cu}$ was significantly higher in the integrated nutrient management treatments. Such positive influence of the application of organic manures in increasing the availability of these nutrients for use by the crops at flowering stage was also reported by Debele et al., (2001) and Reddy (2007).

Effect of different fertility management treatments on grain yield, concentration of $\mathrm{N}, \mathrm{P}, \mathrm{K}, \mathrm{S}, \mathrm{Fe}, \mathrm{Mn}, \mathrm{Zn}$ and $\mathrm{Cu}$ and their uptake by maize grain at harvest

The results (Table 3) showed that the crop was highly sensitive to the reduction in level of $\mathrm{N}$ and $\mathrm{P}$ fertilizers by $50 \%$ of recommended level. The crop fertilized with the recommended level of $120 \mathrm{~kg} \mathrm{~N}$ and $60 \mathrm{~kg}$ $\mathrm{P}_{2} \mathrm{O}_{5}$ per hectare produced $43.10 \mathrm{q} \mathrm{ha}^{-1}$ grain. 
It reduced to $34.23 \mathrm{q} \mathrm{ha}^{-1}$ on application of $50 \%$ of these nutrients through fertilizers. The crop grown without the application of these nutrients produced extremely low yield of $27.26 \mathrm{q} \mathrm{ha}^{-1}$. The substitution of $25 \% \mathrm{~N}$ through poultry manure or vermicompost with or without the addition of Azotobacter increased the grain yield significantly. Such an yield advantage was also recorded by the substitution of $25 \% \mathrm{P}$ fertilizer through the poultry manure or vermicompost with or without the addition of phosphorus solubilising bacteria. Grain yield exceeded 50 $\mathrm{q} \mathrm{ha}{ }^{-1}$ in response to these integrated nutrient management treatments, recording about $7 \mathrm{q}$ $\mathrm{ha}^{-1}$ additional yield compared to the yield obtained through inorganic $\mathrm{N}$ and $\mathrm{P}$.

The uptake of $\mathrm{N}, \mathrm{P} \mathrm{K}$ and $\mathrm{S}$ were not significantly low on application of $75 \% \mathrm{~N}$ and $\mathrm{P}$ than at recommended level. The integrated nutrient management treatments did not induce a significant improvement in the concentration of $\mathrm{N}, \mathrm{P}, \mathrm{K}$ and $\mathrm{S}$. But, their uptake was significant compared to the uptake on application of recommended level of $\mathrm{N}$ and $\mathrm{P}$ through fertilizers.

The results (Table 4) showed that the unfertilized crop had significantly low concentration of $\mathrm{Fe}$ or $\mathrm{Zn}$ in the grain. The concentration of $\mathrm{Mn}$ or $\mathrm{Cu}$ was not influenced by the application of $\mathrm{N}$ and $\mathrm{P}$ through fertilizers. The uptake of these micronutrients was enhanced to a maximum of $78.30 \mathrm{~g} \mathrm{ha}^{-1}$ $\mathrm{Fe}, 63 \mathrm{~g} \mathrm{ha}^{-1} \mathrm{Mn}, 68.5 \mathrm{~g} \mathrm{ha}^{-1} \mathrm{Zn}$ and $58.60 \mathrm{~g}$ ha $^{-1}$

$\mathrm{Cu}$ in response to the application of recommended level of $120 \mathrm{~kg} \mathrm{~N}$ and $60 \mathrm{~kg}$ $\mathrm{P}_{2} \mathrm{O}_{5}$ per hectare. The uptake of all four micronutrients increased significantly by substitution of $25 \% \quad \mathrm{~N}$ or $\mathrm{P}$ through vermicompost or poultry manure with or without the addition of biofertilizers.
Effect of different fertility management treatments on stover yield, concentration of $\mathbf{N}, \mathbf{P}, \mathbf{K}, \mathbf{S}, \mathbf{F e}$

Maize produced $50.46 \mathrm{q} \mathrm{ha}^{-1}$ stover on application of recommended level of $120 \mathrm{~kg}$ $\mathrm{N}$ and $60 \mathrm{~kg} \mathrm{P}_{2} \mathrm{O}_{5} \mathrm{ha}^{-1}$. Each $25 \%$ reduction in level of $\mathrm{N}$ and $\mathrm{P}$ fertilizer successively reduced stover yield. The untreated maize yielded only $25 \mathrm{q} \mathrm{ha}^{-1}$ stover. The substitution of $25 \% \mathrm{~N}$ fertilizer with poultry manure with or without Azotobacter produced stover yield as that obtained on using recommended level of $\mathrm{N}$ and $\mathrm{P}$ through the fertilizers. The substitution of $25 \% \mathrm{~N}$ through vermicompost with or without Azotobacter significantly increased the stover yield to $56.36 \mathrm{q} \mathrm{ha}^{-1}$. The use of poultry manure or vermicompost to substitute $25 \%$ of phosphatic fertilizer increased stover yield significantly. The role of phosphorus solubilising bacteria was not remarkable (Table 5).

The trends in the concentration of N, P K and $\mathrm{S}$ showed considerable variation in response to the reduction in the level of $\mathrm{N}$ and $\mathrm{P}$ applied through fertilizers. The concentration of $\mathrm{N}$ in the stover of maize reduced significantly on reducing the level of $\mathrm{N}$ and $\mathrm{P}$ to $50 \%$ while the $\mathrm{K}$ concentration was on par. The concentration of $\mathrm{P}$ and $\mathrm{S}$ did not change significantly due to reduced $\mathrm{N}$ and $\mathrm{P}$ fertilizers or in control.

The uptake of $\mathrm{N}$ and $\mathrm{K}$ reduced significantly with successive reduction in the level of $\mathrm{N}$ and $\mathrm{P}$ fertilizers. Maize tolerated a moderate reduction in $\mathrm{N}$ and $\mathrm{P}$ fertilizers up to $75 \%$ of the recommended level and recorded no significant reduction in the uptake of $\mathrm{P}$ or $\mathrm{S}$. None of the integrated nutrient management treatments showed a remarkable improvement in the uptake of $\mathrm{N}, \mathrm{P}, \mathrm{K}$ and $\mathrm{S}$ in the stover of maize. All these treatments significantly increased the uptake of $\mathrm{N}$. 
Table.1 Effect of different fertility management treatments on dry matter yield, concentrations (\%) and uptake (kg ha $\left.{ }^{-1}\right)$ of $\mathrm{N}, \mathrm{P}, \mathrm{K}$ and $\mathrm{S}$ at flowering stage (60 DAS) of maize

\begin{tabular}{|c|c|c|c|c|c|c|c|c|c|}
\hline \multirow[t]{2}{*}{ Treatments } & \multirow{2}{*}{$\begin{array}{c}\text { Dry } \\
\text { matter } \\
\text { yield } \\
\left(\mathbf{q} \text { ha }^{-1}\right)\end{array}$} & \multicolumn{2}{|c|}{$\mathbf{N}$} & \multicolumn{2}{|c|}{$\mathbf{P}$} & \multicolumn{2}{|c|}{$\mathbf{K}$} & \multicolumn{2}{|c|}{$\mathbf{S}$} \\
\hline & & $(\%)$ & $\left(\mathrm{kg} \mathrm{ha}^{-1}\right)$ & $(\%)$ & $\left(\mathrm{kg}_{1} \mathrm{ha}^{-}\right.$ & $(\%)$ & (kg ha ${ }^{-}$ & $(\%)$ & $\left(\mathrm{kg} \mathrm{ha}^{-1}\right)$ \\
\hline $\mathrm{T}_{1}$ : Control (No fertilizers) & 30.00 & 1.14 & 34.20 & 0.14 & 4.28 & 0.96 & 28.86 & 0.27 & 8.14 \\
\hline $\mathrm{T}_{2}: 50 \% \mathrm{~N}, \mathrm{P}$ through RDF & 36.83 & 1.20 & 44.19 & 0.16 & 5.71 & 1.07 & 38.98 & 0.28 & 10.19 \\
\hline $\mathrm{T}_{3}: 75 \% \mathrm{~N}, \mathrm{P}$ through RDF & 47.26 & 1.26 & 59.54 & 0.18 & 8.56 & 1.11 & 52.61 & 0.28 & 13.22 \\
\hline $\mathrm{T}_{4}: 100 \% \mathrm{~N}, \mathrm{P}$ through $\mathrm{RDF}\left(120-60 \mathrm{Kg} \mathrm{N}, \mathrm{P}_{2} \mathrm{O}_{5} \mathrm{ha}^{-1}\right)$ & 52.13 & 1.42 & 74.02 & 0.19 & 9.86 & 1.12 & 58.33 & 0.29 & 14.86 \\
\hline $\begin{array}{l}\mathrm{T}_{5}: 75 \% \mathrm{~N} \text { through } \mathrm{RDF}+25 \% \mathrm{~N} \text { through Poultry } \\
\text { manure }\end{array}$ & 55.06 & 1.48 & 81.48 & 0.24 & 13.24 & 1.20 & 66.46 & 0.29 & 15.93 \\
\hline $\begin{array}{l}\mathrm{T}_{6}: \mathbf{7 5 \%} \mathrm{N} \text { through } \mathrm{RDF}+25 \% \mathrm{~N} \text { through Poultry } \\
\text { manure + Azotobacter }\end{array}$ & 56.26 & 1.49 & 83.82 & 0.24 & 13.54 & 1.24 & 69.64 & 0.29 & 16.37 \\
\hline $\begin{array}{l}\mathrm{T}_{7}: 75 \% \mathrm{~N} \text { through } \mathrm{RDF}+25 \% \mathrm{~N} \text { through Vermi } \\
\text { compost }\end{array}$ & 55.40 & 1.50 & 83.10 & 0.24 & 13.37 & 1.20 & 67.01 & 0.29 & 16.12 \\
\hline $\begin{array}{l}\mathrm{T}_{8}: 75 \% \mathrm{~N} \text { through } \mathrm{RDF}+25 \% \mathrm{~N} \text { through } \mathrm{V} . \mathrm{C} .+ \\
\text { AZB }\end{array}$ & 57.10 & 1.51 & 86.22 & 0.25 & 14.23 & 1.18 & 67.89 & 0.29 & 16.67 \\
\hline$T_{9}: 75 \%$ P through RDF + 25\% P through P.M. & 55.26 & 1.48 & 81.78 & 0.24 & 13.24 & 1.20 & 66.25 & 0.29 & 16.05 \\
\hline $\begin{array}{l}\mathrm{T}_{10}: 75 \% \mathrm{P} \text { through RDF }+25 \% \text { P through P.M. + } \\
\text { Phosphorus solubilising bacteria }\end{array}$ & 56.26 & 1.47 & 82.70 & 0.25 & 14.12 & 1.21 & 68.04 & 0.29 & 16.37 \\
\hline$T_{11}: 75 \%$ P through RDF $+25 \%$ P through V.C & 56.33 & 1.50 & 84.49 & 0.25 & 14.04 & 1.19 & 66.93 & 0.29 & 16.39 \\
\hline $\begin{array}{l}\mathrm{T}_{12}: 75 \% \text { P through } \mathrm{RDF}+25 \% \mathrm{P} \text { through } \mathrm{V} . \mathrm{C}+ \\
\text { P.S.B. }\end{array}$ & 56.40 & 1.50 & 84.60 & 0.26 & 14.69 & 1.21 & 68.38 & 0.29 & 16.46 \\
\hline SEm \pm & 2.14 & 0.06 & 1.60 & 0.01 & 0.87 & 0.07 & 4.58 & 0.01 & 0.6 \\
\hline $\mathrm{CD}(\mathrm{P}=0.05)$ & 6.56 & 0.18 & 4.72 & 0.04 & 2.56 & N.S. & 13.53 & N.S. & 1.77 \\
\hline
\end{tabular}


Table.2 Effect of different fertility management treatments on concentrations $\left(\mathrm{mg} \mathrm{kg}^{-1}\right)$ and uptake $\left(\mathrm{g} \mathrm{ha}^{-1}\right)$ of Fe, Mn, $\mathrm{Zn}$ and Cu in drymatter at flowering stage (60 DAS) of maize

\begin{tabular}{|c|c|c|c|c|c|c|c|c|}
\hline \multirow[t]{2}{*}{ Treatments } & \multicolumn{2}{|c|}{$\mathbf{F e}$} & \multicolumn{2}{|c|}{ Mn } & \multicolumn{2}{|c|}{$\mathbf{Z n}$} & \multicolumn{2}{|c|}{$\mathbf{C u}$} \\
\hline & $\begin{array}{l}\text { ( })^{-} \\
\text {kg kg- }\end{array}$ & $\left(\mathrm{g} \mathrm{ha}^{-1}\right)$ & $\begin{array}{l}\left(\mathrm{mg} \mathrm{kg}^{-}\right. \\
\mathbf{1}^{-}\end{array}$ & $\left(\mathrm{g} \mathrm{ha}^{-1}\right)$ & $\begin{array}{r}(\mathrm{mg} \\
\left.\mathrm{kg}^{-1}\right)\end{array}$ & $\left(\mathrm{g} \mathrm{ha}^{-1}\right)$ & $\begin{array}{r}(\mathrm{mg} \\
\left.\mathrm{kg}^{-1}\right)\end{array}$ & $\left(\mathrm{g} \mathrm{ha}^{-1}\right)$ \\
\hline$T_{1}$ : Control (No fertilizers) & 74.0 & 221.9 & 52.3 & 156.9 & 16.9 & 50.6 & 14.8 & 44.4 \\
\hline$T_{2}: 50 \% \mathrm{~N}, \mathrm{P}$ through RDF & 74.5 & 274.4 & 52.4 & 191.8 & 17.7 & 65.6 & 15.4 & 56.4 \\
\hline$T_{3}: 75 \% \mathrm{~N}, \mathrm{P}$ through RDF & 74.9 & 354.3 & 52.5 & 248.3 & 17.7 & 83.7 & 15.5 & 73.1 \\
\hline $\mathrm{T}_{4}: 100 \% \mathrm{~N}, \mathrm{P}$ through $\mathrm{RDF}\left(120-60 \mathrm{Kg} \mathrm{N}, \mathrm{P}_{2} \mathrm{O}_{5} \mathrm{ha}^{-1}\right)$ & 75.3 & 392.5 & 52.9 & 275.5 & 17.9 & 93.4 & 15.6 & 81.2 \\
\hline $\begin{array}{l}\mathrm{T}_{5}: 75 \% \mathrm{~N} \text { through } \mathrm{RDF}+25 \% \mathrm{~N} \text { through Poultry } \\
\text { manure }\end{array}$ & 77.2 & 425.1 & 56.7 & 312.2 & 18.3 & 100.7 & 16.2 & 89.2 \\
\hline $\begin{array}{l}\mathrm{T}_{6}: \mathbf{7 5 \%} \mathrm{N} \text { through } \mathrm{RDF}+25 \% \mathrm{~N} \text { through Poultry } \\
\text { manure }+ \text { Azotobacter }\end{array}$ & 77.5 & 436.0 & 56.8 & 319.6 & 18.4 & 103.4 & 16.4 & 92.3 \\
\hline $\begin{array}{l}\mathrm{T}_{7}: 75 \% \mathrm{~N} \text { through } \mathrm{RDF}+25 \% \mathrm{~N} \text { through Vermi } \\
\text { compost }\end{array}$ & 77.8 & 431.0 & 57.2 & 317.4 & 18.6 & 10.3 .2 & 16.5 & 91.4 \\
\hline $\begin{array}{l}\mathrm{T}_{8}: 75 \% \mathrm{~N} \text { through } \mathrm{RDF}+25 \% \mathrm{~N} \text { through } \mathrm{V} . \mathrm{C} .+ \\
\text { AZB }\end{array}$ & 78.0 & 445.3 & 57.5 & 328.3 & 18.8 & 107.2 & 16.8 & 95.9 \\
\hline$T_{9}: 75 \%$ P through RDF $+25 \%$ P through P.M. & 77.6 & 428.8 & 56.8 & 313.9 & 18.4 & 101.7 & 16.6 & 92.0 \\
\hline $\begin{array}{l}\mathrm{T}_{10}: 75 \% \text { P through RDF }+25 \% \text { P through P.M. + } \\
\text { Phosphorus solubilising bacteria }\end{array}$ & 77.8 & 437.7 & 57.2 & 321.5 & 18.5 & 104.0 & 16.7 & 93.9 \\
\hline$T_{11}: 75 \%$ P through RDF $+25 \%$ P through V.C & 77.8 & 438.2 & 57.2 & 322.2 & 18.4 & 103.6 & 16.4 & 92.6 \\
\hline $\begin{array}{l}\text { T12: }_{15 \%} \text { P through RDF }+25 \% \text { P through V.C }+ \\
\text { P.S.B. }\end{array}$ & 77.9 & 439.6 & 57.3 & 323.1 & 18.6 & 104.9 & 16.8 & 94.7 \\
\hline SEm \pm & 0.33 & 17.22 & 0.69 & 11.81 & 0.33 & 4.67 & 0.18 & 3.53 \\
\hline $\mathrm{CD}(\mathrm{P}=0.05)$ & 1.00 & 50.8 & 2.1 & 34.9 & 0.98 & 13.7 & 0.53 & 10.4 \\
\hline
\end{tabular}


Table.3 Effect of different fertility management treatments on grain yield, concentrations (\%) and uptake $\left(\mathrm{kg} \mathrm{ha}^{-1}\right)$ of $\mathrm{N}, \mathrm{P}, \mathrm{K}$ and $\mathrm{S}$ at harvest of maize (Field experiment during kharif, 2009)

\begin{tabular}{|c|c|c|c|c|c|c|c|c|c|}
\hline \multirow[t]{2}{*}{ Treatments } & \multirow{2}{*}{$\begin{array}{c}\text { Grain } \\
\text { yield } \\
\left(\mathbf{q ~ h a} \mathbf{h}^{-1}\right)\end{array}$} & \multicolumn{2}{|r|}{$\mathbf{N}$} & \multicolumn{2}{|c|}{$\mathbf{P}$} & \multicolumn{2}{|c|}{$\mathbf{K}$} & \multicolumn{2}{|c|}{$\mathbf{S}$} \\
\hline & & $(\%)$ & $\left(\mathrm{kg} \mathrm{ha}^{-1}\right)$ & $(\%)$ & $\left(\mathrm{kg} \mathrm{ha}^{-1}\right)$ & $(\%)$ & $\left(\mathrm{kg} \mathrm{ha}^{-1}\right)$ & $(\%)$ & $\left(\mathrm{kg} \mathrm{ha}^{-1}\right)$ \\
\hline$T_{1}:$ Control (No fertilizers) & 27.26 & 1.20 & 32.99 & 0.15 & 4.13 & 0.65 & 17.75 & 0.26 & 7.11 \\
\hline $\mathrm{T}_{2}: 50 \% \mathrm{~N}, \mathrm{P}$ through RDF & 34.23 & 1.25 & 42.99 & 0.20 & 6.94 & 0.69 & 23.52 & 0.27 & 9.28 \\
\hline$T_{3}: 75 \% \mathrm{~N}, \mathrm{P}$ through RDF & 39.16 & 1.33 & 52.15 & 0.22 & 8.73 & 0.72 & 28.36 & 0.27 & 10.61 \\
\hline $\mathrm{T}_{4}: 100 \% \mathrm{~N}, \mathrm{P}$ through $\mathrm{RDF}\left(120-60 \mathrm{Kg} \mathrm{N}, \mathrm{P}_{2} \mathrm{O}_{5} \mathrm{ha}^{-1}\right)$ & 43.10 & 1.34 & 58.24 & 0.24 & 10.42 & 0.75 & 32.18 & 0.28 & 12.12 \\
\hline $\begin{array}{l}\mathrm{T}_{5}: 75 \% \mathrm{~N} \text { through } \mathrm{RDF}+25 \% \mathrm{~N} \text { through Poultry } \\
\text { manure }\end{array}$ & 50.23 & 1.38 & 69.73 & 0.27 & 13.46 & 0.77 & 38.82 & 0.29 & 14.52 \\
\hline $\begin{array}{l}\mathrm{T}_{6}: 75 \% \mathrm{~N} \text { through } \mathrm{RDF}+25 \% \mathrm{~N} \text { through Poultry } \\
\text { manure + Azotobacter }\end{array}$ & 51.26 & 1.41 & 72.66 & 0.28 & 14.27 & 0.78 & 40.06 & 0.29 & 14.82 \\
\hline $\begin{array}{l}\mathrm{T}_{7}: 75 \% \mathrm{~N} \text { through } \mathrm{RDF}+25 \% \mathrm{~N} \text { through Vermi } \\
\text { compost }\end{array}$ & 52.26 & 1.42 & 74.16 & 0.28 & 14.67 & 0.79 & 41.35 & 0.29 & 15.17 \\
\hline $\begin{array}{l}\text { T }_{8}: 75 \% \mathrm{~N} \text { through RDF }+25 \% \mathrm{~N} \text { through V.C. }+ \\
\text { AZB }\end{array}$ & 53.23 & 1.44 & 77.15 & 0.29 & 15.41 & 0.80 & 42.68 & 0.30 & 15.92 \\
\hline$T_{9}: 75 \% P$ through RDF $+25 \%$ P through P.M. & 50.26 & 1.40 & 70.58 & 0.28 & 14.12 & 0.78 & 39.19 & 0.29 & 14.61 \\
\hline $\begin{array}{l}\mathrm{T}_{10}: 75 \% \mathrm{P} \text { through } \mathrm{RDF}+25 \% \mathrm{P} \text { through P.M. }+ \\
\text { Phosphorus solubilising bacteria }\end{array}$ & 52.20 & 1.41 & 73.51 & 0.29 & 15.09 & 0.79 & 41.21 & 0.29 & 15.09 \\
\hline $\mathrm{T}_{11}: 75 \% \mathrm{P}$ through RDF $+25 \% \mathrm{P}$ through V.C & 51.53 & 1.41 & 72.73 & 0.28 & 14.38 & 0.80 & 41.25 & 0.29 & 14.91 \\
\hline $\begin{array}{l}\mathrm{T}_{12}: 75 \% \mathrm{P} \text { through } \mathrm{RDF}+25 \% \mathrm{P} \text { through } \mathrm{V} . \mathrm{C}+ \\
\text { P.S.B. }\end{array}$ & 53.10 & 1.42 & 75.64 & 0.29 & 15.34 & 0.80 & 42.49 & 0.30 & 15.90 \\
\hline SEm \pm & 1.41 & 0.04 & 3.10 & 0.02 & 1.05 & 0.03 & 1.93 & 0.01 & 0.66 \\
\hline $\mathrm{CD}(\mathrm{P}=\mathbf{0 . 0 5})$ & 4.18 & 0.13 & 9.17 & 0.07 & 3.12 & 0.08 & 5.69 & N.S. & 1.95 \\
\hline
\end{tabular}


Table.4 Effect of different fertility management treatments on concentration $\left(\mathrm{mg} \mathrm{kg}^{-1}\right)$ and uptake $\left(\mathrm{g} \mathrm{ha}^{-1}\right)$ of Fe $\mathrm{Mn}, \mathrm{Zn}$ and Cu in maize grain at harvest of maize

\begin{tabular}{|c|c|c|c|c|c|c|c|c|}
\hline \multirow[t]{2}{*}{ Treatments } & \multicolumn{2}{|c|}{ Fe } & \multicolumn{2}{|c|}{ Mn } & \multicolumn{2}{|c|}{$\mathbf{Z n}$} & \multicolumn{2}{|c|}{$\mathbf{C u}$} \\
\hline & $\left(\mathrm{mg} \mathrm{kg}^{-}\right.$ & (g ha ${ }^{-}$ & 1) ${ }^{\left(\mathrm{mg} \mathrm{kg}^{-}\right.}$ & (g ha- & ( $)^{\left(\mathrm{mg} \mathrm{kg}^{-}\right.}$ & (g ha ${ }^{-}$ & $\left(\mathrm{mg} \mathrm{kg}^{-}\right.$ & $\left(\mathrm{gha}^{-1}\right)$ \\
\hline$T_{1}:$ Control (No fertilizers) & 17.30 & 47.20 & 14.30 & 39.00 & 15.70 & 42.80 & 13.20 & 35.90 \\
\hline $\mathrm{T}_{2}: 50 \% \mathrm{~N}, \mathrm{P}$ through RDF & 17.60 & 60.00 & 14.40 & 49.10 & 15.80 & 54.00 & 13.30 & 45.50 \\
\hline $\mathrm{T}_{3}: \mathbf{7 5 \%} \mathrm{N}, \mathrm{P}$ through RDF & 17.80 & 69.90 & 14.40 & 56.40 & 15.80 & 61.80 & 13.40 & 52.40 \\
\hline $\mathrm{T}_{4}: 100 \% \mathrm{~N}, \mathrm{P}$ through $\mathrm{RDF}\left(120-60 \mathrm{Kg} \mathrm{N}, \mathrm{P}_{2} \mathrm{O}_{5} \mathrm{ha}^{-1}\right)$ & 18.20 & 78.30 & 14.60 & 63.00 & 15.90 & 68.50 & 13.60 & 58.60 \\
\hline $\begin{array}{l}\mathrm{T}_{5}: 75 \% \mathrm{~N} \text { through } \mathrm{RDF}+25 \% \mathrm{~N} \text { through Poultry } \\
\text { manure }\end{array}$ & 19.30 & 96.80 & 15.20 & 76.10 & 16.80 & 84.50 & 14.50 & 72.70 \\
\hline $\begin{array}{l}\mathrm{T}_{6}: 75 \% \mathrm{~N} \text { through } \mathrm{RDF}+25 \% \mathrm{~N} \text { through Poultry } \\
\text { manure + Azotobacter }\end{array}$ & 19.40 & 99.30 & 15.40 & 79.10 & 16.80 & 86.40 & 14.60 & 74.80 \\
\hline $\begin{array}{l}\mathrm{T}_{7}: 75 \% \mathrm{~N} \text { through } \mathrm{RDF}+25 \% \mathrm{~N} \text { through Vermi } \\
\text { compost }\end{array}$ & 19.40 & 101.40 & 15.60 & 81.50 & 16.90 & 88.40 & 14.80 & 77.30 \\
\hline $\begin{array}{l}\mathrm{T}_{8}: 75 \% \mathrm{~N} \text { through } \mathrm{RDF}+25 \% \mathrm{~N} \text { through } \mathrm{V} . \mathrm{C} .+ \\
\text { AZB }\end{array}$ & 19.60 & 104.30 & 15.80 & 83.90 & 17.10 & 91.10 & 14.90 & 79.30 \\
\hline $\mathrm{T}_{9}: 75 \% \mathrm{P}$ through RDF $+25 \%$ P through P.M. & 19.30 & 97.00 & 15.50 & 77.90 & 16.80 & 84.60 & 14.60 & 73.30 \\
\hline $\begin{array}{l}\mathrm{T}_{10}: 75 \% \text { P through RDF }+25 \% \text { P through P.M. + } \\
\text { Phosphorus solubilising bacteria }\end{array}$ & 19.40 & 101.20 & 15.60 & 81.30 & 16.90 & 88.20 & 14.70 & 76.70 \\
\hline$T_{11}: 75 \%$ P through RDF $+25 \%$ P through V.C & 19.50 & 100.40 & 15.60 & 80.40 & 16.90 & 87.10 & 14.60 & 75.20 \\
\hline $\begin{array}{l}T_{12}: 75 \% \text { P through RDF }+25 \% \text { P through V.C + } \\
\text { P.S.B. }\end{array}$ & 19.60 & 104.10 & 15.80 & 83.90 & 17.00 & 90.40 & 14.70 & 78.00 \\
\hline SEm \pm & 0.25 & 2.65 & 0.36 & 2.38 & 0.05 & 1.93 & 0.13 & 1.59 \\
\hline $\mathrm{CD}(\mathrm{P}=\mathbf{0 . 0 5})$ & 0.75 & 7.82 & 1.06 & 7.03 & 0.15 & 5.70 & 0.40 & 4.72 \\
\hline
\end{tabular}


Table.5 Effect of different fertility management treatments on stover yield, concentrations (\%) and uptake $\left(\mathrm{kg} \mathrm{ha}{ }^{-1}\right)$ of $\mathrm{N}, \mathrm{P}, \mathrm{K}$ and $\mathrm{S}$ at harvest of maize

\begin{tabular}{|c|c|c|c|c|c|c|c|c|c|}
\hline \multirow[t]{2}{*}{ Treatments } & \multirow{2}{*}{$\begin{array}{c}\text { Stover } \\
\text { yield } \\
\left(\mathbf{q} \mathbf{h a}^{-1}\right)\end{array}$} & \multicolumn{2}{|c|}{$\mathbf{N}$} & \multicolumn{2}{|c|}{$\mathbf{P}$} & \multicolumn{2}{|c|}{$\mathbf{K}$} & \multicolumn{2}{|c|}{$\mathbf{S}$} \\
\hline & & $(\%)$ & $\left(\mathbf{k g ~ h a}^{-}\right.$ & $(\%)$ & $\underset{1}{\left(k_{g} \mathbf{h a}^{-}\right.}$ & $(\%)$ & $\left(\mathbf{k g ~ h a}^{-}\right.$ & $(\%)$ & $\begin{array}{r}(\mathbf{k g} \\
\left.\mathbf{h a}^{-1}\right)\end{array}$ \\
\hline$T_{1}:$ Control (No fertilizers) & 25.00 & 0.60 & 14.93 & 0.12 & 2.99 & 1.04 & 25.98 & 0.20 & 4.99 \\
\hline $\mathrm{T}_{2}: 50 \% \mathrm{~N}, \mathrm{P}$ through RDF & 36.36 & 0.65 & 23.58 & 0.13 & 4.76 & 1.06 & 38.62 & 0.21 & 7.61 \\
\hline $\mathrm{T}_{3}: 75 \% \mathrm{~N}, \mathrm{P}$ through $\mathrm{RDF}$ & 44.23 & 0.70 & 31.09 & 0.14 & 6.27 & 1.11 & 49.21 & 0.21 & 9.36 \\
\hline $\mathrm{T}_{4}: 100 \% \mathrm{~N}, \mathrm{P}$ through $\mathrm{RDF}\left(120-60 \mathrm{Kg} \mathrm{N}, \mathrm{P}_{2} \mathrm{O}_{5} \mathrm{ha}^{-1}\right)$ & 50.46 & 0.79 & 39.75 & 0.15 & 7.61 & 1.12 & 56.70 & 0.22 & 11.13 \\
\hline $\begin{array}{l}\mathrm{T}_{5}: 75 \% \mathrm{~N} \text { through } \mathrm{RDF}+25 \% \mathrm{~N} \text { through Poultry } \\
\text { manure }\end{array}$ & 54.13 & 0.85 & 46.66 & 0.18 & 9.77 & 1.12 & 60.65 & 0.23 & 12.41 \\
\hline $\begin{array}{l}\mathrm{T}_{6}: \mathbf{7 5 \%} \mathrm{N} \text { through } \mathrm{RDF}+25 \% \mathrm{~N} \text { through Poultry } \\
\text { manure + Azotobacter }\end{array}$ & 54.43 & 0.86 & 46.75 & 0.19 & 10.36 & 1.13 & 61.55 & 0.23 & 12.54 \\
\hline $\begin{array}{l}\mathrm{T}_{7}: 75 \% \mathrm{~N} \text { through } \mathrm{RDF}+25 \% \mathrm{~N} \text { through Vermi } \\
\text { compost }\end{array}$ & 55.30 & 0.87 & 48.12 & 0.18 & 9.93 & 1.15 & 63.61 & 0.24 & 13.28 \\
\hline $\mathrm{T}_{8}: \mathbf{7 5 \%} \mathrm{N}$ through $\mathrm{RDF}+25 \% \mathrm{~N}$ through $\mathrm{VC}+\mathrm{AZB}$ & 56.36 & 0.90 & 50.70 & 0.20 & 11.22 & 1.16 & 65.34 & 0.24 & 13.50 \\
\hline$T_{9}: 75 \%$ P through RDF + 25\% P through PM & 55.10 & 0.86 & 47.44 & 0.19 & 10.53 & 1.13 & 62.05 & 0.23 & 12.63 \\
\hline $\begin{array}{l}\mathrm{T}_{10}: 75 \% \mathrm{P} \text { through } \mathrm{RDF}+25 \% \mathrm{P} \text { through } \mathrm{PM}+ \\
\text { Phosphorus solubilising bacteria }\end{array}$ & 56.26 & 0.89 & 50.10 & 0.21 & 11.78 & 1.15 & 64.67 & 0.23 & 13.12 \\
\hline$T_{11}: 75 \%$ P through RDF + 25\% P through VC & 55.16 & 0.86 & 47.49 & 0.19 & 10.53 & 1.15 & 63.52 & 0.23 & 12.76 \\
\hline$T_{12}: 75 \% P$ through RDF $+25 \% P$ through $V C+P S B$ & 56.23 & 0.89 & 49.99 & 0.22 & 12.31 & 1.16 & 65.27 & 0.24 & 13.48 \\
\hline SEm \pm & 1.36 & 0.03 & 1.17 & 0.02 & 0.95 & 0.02 & 2.03 & 0.01 & 0.75 \\
\hline $\mathrm{CD}(\mathrm{P}=0.05)$ & 4.03 & 0.10 & 3.47 & 0.05 & 2.80 & 0.06 & 6.01 & N.S. & 2.20 \\
\hline
\end{tabular}


Table.6 Effect of different fertility management treatments on concentrations $\left(\mathrm{mg} \mathrm{kg}^{-1}\right)$ and uptake $\left(\mathrm{g} \mathrm{ha}^{-1}\right)$ of Fe, $\mathrm{Mn}, \mathrm{Zn}$ and Cu in stover of maize at harvest

\begin{tabular}{|c|c|c|c|c|c|c|c|c|}
\hline \multirow[t]{2}{*}{ Treatments } & \multicolumn{2}{|c|}{$\mathbf{F e}$} & \multicolumn{2}{|c|}{ Mn } & \multicolumn{2}{|c|}{$\mathbf{Z n}$} & \multicolumn{2}{|c|}{$\mathbf{C u}$} \\
\hline & $\underset{1}{\left(\mathrm{mg} \mathrm{kg}^{-}\right.}$ & $\left(\mathrm{g} \mathrm{ha}^{-}\right.$ & $\underset{1}{\left(\mathrm{mg} \mathrm{kg}^{-}\right.}$ & $\left(\mathrm{g} \mathrm{ha}^{-}\right.$ & $(\mathbf{m g})$ & $\left(\mathrm{g} \mathrm{ha}^{-}\right.$ & $(\mathbf{m g}$ & $\left(\mathrm{g} \mathrm{ha}^{-1}\right)$ \\
\hline$T_{1}:$ Control (No fertilizers) & 41.2 & 102.9 & 32.6 & 81.4 & 16.2 & 40.4 & 14.1 & 35.2 \\
\hline $\mathrm{T}_{2}: 50 \% \mathrm{~N}, \mathrm{P}$ through RDF & 42.1 & 153.4 & 34.2 & 124.1 & 16.3 & 59.2 & 14.3 & 52.0 \\
\hline$T_{3}: 75 \% \mathrm{~N}, \mathrm{P}$ through $\mathrm{RDF}$ & 42.1 & 186.7 & 34.8 & 154.1 & 16.3 & 72.0 & 14.3 & 63.3 \\
\hline $\mathrm{T}_{4}: 100 \% \mathrm{~N}, \mathrm{P}$ through $\mathrm{RDF}\left(120-60 \mathrm{Kg} \mathrm{N}, \mathrm{P}_{2} \mathrm{O}_{5} \mathrm{ha}^{-1}\right)$ & 42.6 & 214.9 & 34.9 & 176.0 & 16.8 & 84.7 & 14.4 & 72.9 \\
\hline $\begin{array}{l}\mathrm{T}_{5}: 75 \% \mathrm{~N} \text { through } \mathrm{RDF}+25 \% \mathrm{~N} \text { through Poultry } \\
\text { manure }\end{array}$ & 43.2 & 233.8 & 35.2 & 190.5 & 16.9 & 91.7 & 15.4 & 83.5 \\
\hline $\begin{array}{l}\mathrm{T}_{6}: 75 \% \mathrm{~N} \text { through } \mathrm{RDF}+25 \% \mathrm{~N} \text { through Poultry } \\
\text { manure + Azotobacter }\end{array}$ & 43.4 & 236.2 & 35.4 & 192.6 & 17.0 & 92.9 & 15.5 & 84.0 \\
\hline $\begin{array}{l}\mathrm{T}_{7}: 75 \% \mathrm{~N} \text { through } \mathrm{RDF}+25 \% \mathrm{~N} \text { through Vermi } \\
\text { compost }\end{array}$ & 43.1 & 238.7 & 35.5 & 196.3 & 17.1 & 94.9 & 15.5 & 85.6 \\
\hline$T_{8}: 75 \% \mathrm{~N}$ through $\mathrm{RDF}+25 \% \mathrm{~N}$ through $\mathrm{V} . \mathrm{C} .+\mathrm{AZB}$ & 43.6 & 245.8 & 35.6 & 200.7 & 17.1 & 96.5 & 15.5 & 87.3 \\
\hline T. $_{9}: 75 \%$ P through RDF $+25 \%$ P through P.M. & 43.3 & 238.6 & 35.4 & 194.9 & 16.8 & 92.8 & 15.4 & 85.1 \\
\hline $\begin{array}{l}\mathrm{T}_{10}: 75 \% \mathrm{P} \text { through } \mathrm{RDF}+25 \% \mathrm{P} \text { through P.M. }+ \\
\text { Phosphorus solubilising bacteria }\end{array}$ & 43.4 & 244.1 & 35.5 & 199.6 & 16.9 & 95.0 & 15.4 & 87.1 \\
\hline $\mathrm{T}_{11}: 75 \% \mathrm{P}$ through RDF $+25 \%$ P through V.C & 43.4 & 239.3 & 35.5 & 195.8 & 17.1 & 94.3 & 15.4 & 85.0 \\
\hline$T_{12}: 75 \%$ P through RDF $+25 \%$ P through V.C + P.S.B. & 43.5 & 244.5 & 35.6 & 199.9 & 17.2 & 96.4 & 15.5 & 87.0 \\
\hline SEm \pm & 0.69 & 6.52 & 0.41 & 4.48 & 0.07 & 2.12 & 0.05 & 2.09 \\
\hline $\mathrm{CD}(\mathrm{P}=0.05)$ & 1.44 & 19.3 & 1.23 & 13.2 & 0.22 & 6.27 & 0.13 & 6.18 \\
\hline
\end{tabular}


The substitution of $25 \% \mathrm{~N}$ fertilizer with poultry manure did not increase $\mathrm{P}, \mathrm{K}$ or $\mathrm{S}$ in the stover. The uptake of $\mathrm{P}$ and $\mathrm{K}$ increased significantly by substituting $25 \% \mathrm{~N}$ fertilizer with vermicompost and the addition of Azotobacter or the substitution of $25 \% \mathrm{P}$ fertilizer with poultry manure or vermicompost supplemented with the biofertilizers or not. The integration of vermicmpost to substitute $25 \% \mathrm{~N}$ fertilizer with the addition of biofertilizers was the only treatment to significantly increase the uptake of $\mathrm{S}$ by maize stover.

The uptake of $\mathrm{Fe}$ did not increase significantly on increasing levels of $\mathrm{N}$ and $\mathrm{P}$ through fertilizers up to their recommended level (Table 6). The uptake of Mn increased significantly on application of different levels of $\mathrm{N}$ and $\mathrm{P}$ fertilizers. The $\mathrm{Zn}$ uptake showed a significant response only in case of recommended level of $120 \mathrm{~kg} \mathrm{~N}$ and $60 \mathrm{~kg}$ $\mathrm{P}_{2} \mathrm{O}_{5} \mathrm{ha}^{-1}$ and decreased at lower levels. The uptake of $\mathrm{Cu}$ was more on application of recommended level of $\mathrm{N}$ and $\mathrm{P}$ fertilizers than control. The stover removed $214.9 \mathrm{~g} \mathrm{ha}^{-1} \mathrm{Fe}$, $176.0 \mathrm{~g} \mathrm{ha}^{-1} \mathrm{Mn}, 84.7 \mathrm{~g} \mathrm{ha}^{-1} \mathrm{Zn}$ and $72.9 \mathrm{~g} \mathrm{ha}^{-}$ ${ }^{1} \mathrm{Cu}$ on application of recommended level of $\mathrm{N}$ and $\mathrm{P}$. The uptake of these micronutrients reduced at lower levels of $\mathrm{N}$ and $\mathrm{P}$ fertilizers. The Fe and Mn concentration of maize stover due to different integrated nutrient management practices was on par with recommended level of $\mathrm{N}$ and $\mathrm{P}$ fertilizers. The $\mathrm{Zn}$ concentration recorded significant improvement by substituting $25 \% \mathrm{~N}$ with vermicompost with or without the addition of Azotobacter and also by substituting $25 \% \mathrm{P}$ fertilizer with vermicompost and addition of phosphorus solubilising bacteria. The concentration of $\mathrm{Cu}$ recorded a significant increase in maize stover on adoption of any one of the integrated nutrient management practices. The uptake of all the micronutrients was significantly higher due to the integrated nutrient management treatments compared to inorganic fertilizer application.

The results of effect of fertility treatments on grain, stover yield and nutrient content and uptake are discussed here. The improvement in the nutrient concentration in the grain is an indication of its nutritive value for consumption as a staple food. This is also an important consideration in case of poultry feed as maize grain forms the main ingredient, this determines the health of the birds. The nutrient concentration in the stover is also an important determining factor of the nutrition value of feed. Nutrient enrichment of fodder is important in fodder maize as it influences lactogenic property. The nutrient uptake by maize showed higher nutrient requirement for high yield (Singh and Totawat, 2002 and Prasad et al., 2005). The present investigation showed that the maize grain had twice the concentration of $\mathrm{N}$ and 1.5 times the concentration of $\mathrm{P}$ than the stover. The concentration of sulfur was also more in grain than in fodder on the other hand the leaves were rich in $\mathrm{K}$ than the grain.

The concentration of micronutrients i.e. Fe, $\mathrm{Mn}$ and $\mathrm{Cu}$ was more in the stover, while the concentration of $\mathrm{Zn}$ was equal both in the grain and stover. Li et al., (2007) reported that the transfer of micronutrients from the straw to grain is a slow process. Probably this is the reason that the micronutrient concentration of the grain was less.

Hence it is concluded that integrated nutrient management in maize is advantageous to the farmer in improving the quality of grain and fodder as well as in increase in productivity.

\section{References}

Anita, B., Barik, K. C., Garnayak, L. M and Mahapatra 2007. Nitrogen management in baby corn (Zea mays). Indian Journal of Agronomy. 52(2):135-138. 
Debele, T., Sharnappa., Sudhir, K and Sujith, G. M. 2001. Direct and interactive effects of enriched FYM and nitrogen levels on the productivity and nutrient uptake of maize. Karnataka Journal of Agricultural Sciences. 14(4) : 894-899.

Li, B. Y., Zhou, D. M., Cang, L., Zhang, H. L., Fan, X. H and Qin, S. W. 2007. Soil micronutrient availability to crops as affected by long-term inoraganic and organic fertilizer applications. Soil and Tillage Research. 96:166-173.

Panwar, A. S and Munda, G. C. 2006. Response of babycorn (Zea mays L.) to nitrogen and land configuration in mid hills of Meghalaya. The Indian Journal of Agricultural Sciences. 76(5) : 293296.

Prasad, P. R. K., Radha Krishna, Y., Anitha, G., Uma Mahesh, M. N. V. A and Sankar Rao, V. 2005. Effect of different organic manures on dehydrogenase activity in salt affected soils of coastal Andhra Pradesh. The Andhra Agricultural Journal. 52(1\&2) : 147-
149.

Reddy, K. P. 2007. Effect of integrated use of inorganic and organic and inorganic sources of nutrients in maize-groundnut cropping system on Alfisols. Ph.D Thesis.

Singh, R and Totawat, K.L. 2002. Effect of integrated use of nitrogen on the performance of maize (Zea mays L.) on haplustalfs of sub-humid southern plains of Rajasthan. Indian journal of Agricultural Research. 36(2): 102-107.

Srikanth, M., Mohammed Amanullah, M and Muthukrishnan, P. 2009. Influence of plant density and fertilizer on yield attributes, yield and grain quality of hybrid maize.

Verma, C. P., Kedar Prasad., Singh, H. V and Verma, R. N. 2003. Effect of soil conditioners and fertilizers on yield and economics of maize (Zea mays L.) in maize - wheat sequence. Crop Research. 25(3) : 449-453.

\section{How to cite this article:}

Venkata Subbaiah, P. and Sai Ram, A. 2019. Effect of Integrated Use of Organic and Inorganic Sources of Nutrients and Biofertilizers on Growth and Nutrient Content of Maize (Zea mays L.). Int.J.Curr.Microbiol.App.Sci. 8(07): 1106-1116. doi: https://doi.org/10.20546/ijcmas.2019.807.131 